\title{
The ATF6 branch of unfolded protein response and apoptosis are activated to promote African swine fever virus infection
}

\author{
I Galindo ${ }^{1}$, B Hernáez ${ }^{1}$, R Muñoz-Moreno ${ }^{1}$, MA Cuesta-Geijo ${ }^{1}$, I Dalmau-Mena ${ }^{1}$ and C Alonso ${ }^{\star, 1}$
}

\begin{abstract}
African swine fever virus (ASFV) infection induces apoptosis in the infected cell; however, the consequences of this activation on virus replication have not been defined. In order to identify the role of apoptosis in ASFV infection, we analyzed caspase induction during the infection and the impact of caspase inhibition on viral production. Caspases 3,9 and 12 were activated from $16 \mathrm{~h}$ post-infection, but not caspase 8 . Indeed, caspase 3 activation during the early stages of the infection appeared to be crucial for efficient virus exit. In addition, the inhibition of membrane blebbing reduced the release of virus particles from the cell. ASFV uses the endoplasmic reticulum (ER) as a site of replication and this process can trigger ER stress and the unfolded protein response (UPR) of the host cell. In addition to caspase 12 activation, indicators of ER stress include the upregulation of the chaperones calnexin and calreticulin upon virus infection. Moreover, ASFV induces transcription factor 6 signaling pathway of the UPR, but not the protein kinase-like ER kinase or the inositol-requiring enzyme 1 pathways. Thus, the capacity of ASFV to regulate the UPR may prevent early apoptosis and ensure viral replication.
\end{abstract}

Cell Death and Disease (2012) 3, e341; doi:10.1038/cddis.2012.81; published online 5 July 2012

Subject Category: Experimental Medicine

African swine fever virus (ASFV) is a double-stranded large DNA virus that induces an acute disease of swine in which apoptosis has a central role in pathogenesis. Virus infection elicits apoptosis in target and immune defense cells. ${ }^{1}$ The large repertoire of anti-apoptotic proteins encoded by the virus reflects the relevance of apoptosis in limiting viral replication in host cells. Many DNA viruses encode structural and functional homologs of anti-apoptotic Bcl-2 proteins, named viral Bcl2, including the $A 179 L$ gene of $A S F V .{ }^{2}$ In common with other viruses, ASFV has several strategies to block apoptotic pathways in order to complete virus replication. ASFV encodes proteins involved in apoptosis inhibition, thereby delaying the final execution step of the apoptotic pathway, which occurs only at late post-infection times. Programmed cell death itself may be relevant for the final release of virus particles within apoptotic bodies, which would provide this virus with a mechanism to spread, thus evading the immune system. The central component of the apoptotic machinery is the caspase proteolytic system. There are two groups of caspases: upstream initiator caspases, such as caspases 8 and 9, which cleave and activate other caspases; and downstream effector caspases, including caspase 3, which cleave a variety of cellular substrates, thereby disassembling cellular structures or inactivating enzymes. ${ }^{3}$
Caspase 12 is associated with the cytoplasmic face of the endoplasmic reticulum (ER) and it cleaves to an active form in response to ER stress. ${ }^{4}$ The ER stress response, also called the unfolded protein response (UPR), is mediated by three transmembrane proteins: (i) the protein kinase-like ER resident kinase (PERK); (ii) the activating transcription factor 6 (ATF6); and (iii) the inositol-requiring enzyme 1 (IRE1). ${ }^{5}$ These three proteins are associated with the ER chaperone $\mathrm{BiP} / \mathrm{Grp78}$, which prevents their aggregation and further activation. However, when misfolded proteins accumulate, $\mathrm{BiP}$ is released, thus allowing UPR activation. Activated PERK phosphorylates the eukaryotic initiation factor $2 \alpha$ (elF2 $\alpha$ ), thus resulting in translation attenuation to counterbalance enhanced protein accumulation. This counterbalancing effect includes the upregulation of the pro-apoptotic mRNA CHOP (c/EBP homologous protein) and mRNA encoding GADD34 (growth arrest and DNA-damage-inducible protein-34), whose association with the protein phosphatase 1 (PP1) leads to the dephosphorylation of elF2 $\alpha .^{5}$ Activated IRE1 removes 26 nucleotides from $X$-box binding protein-1 (XBP1) mRNA, thereby altering the open reading frame and causing the translation of an active transcription factor. ${ }^{6}$ The spliced form of XBP1 protein (sXBP1) is involved in the transcriptional activation of a number of genes,

\footnotetext{
${ }^{1}$ Departamento de Biotecnología, Instituto Nacional de Investigación y Tecnología Agraria y Alimentaria (INIA), Madrid, Spain

${ }^{*}$ Corresponding author: C Alonso, Departamento de Biotecnología, Instituto Nacional de Investigación y Tecnología Agraria y Alimentaria (INIA), Ctra. de la Coruña Km 7.5, Madrid 28040, Spain. Tel: +34 91347 6896; Fax: +34 91347 8771; E-mail: calonso@inia.es

Keywords: unfolded protein response; ER stress; ATF6; chaperones; caspase 12; African swine fever virus

Abbreviations: ATF6, activating transcription factor 6; ASFV, African swine fever virus; UPR, unfolded protein response; ER, endoplasmic reticulum; PERK, protein kinase-like ER resident kinase; IRE1, inositol-requiring enzyme 1; BiP/Grp78, binding immunoglobulin protein/glucose-regulated protein 78; CHOP/GADD153, c/EBP homologous protein/DNA-damage-inducible gene 153; elF2 $\alpha$, eukaryotic initiation factor 2 $\alpha$; GADD34, growth arrest and DNA-damage-inducible protein-34; PP1, protein phosphatase 1; XBP1, X-box binding Protein-1; EDEM, ER degradation enhancing $\alpha$-mannosidase-like protein; HCV, hepatitis C virus; BVDV, bovine viral diarrhea virus; JEV, Japanese encephalitis virus; CMV, cytomegalovirus; SARS-CoV, severe acute respiratory syndrome virus; AEBSF, 4-(2-aminoethyl)benzenesulfonyl fluoride

Received 08.12.11; revised 10.5.12; accepted 29.5.12; Edited by M Piacentini
} 
including the ER mannosidase-like protein EDEM, which participates in protein degradation. Finally, activated ATF6 exits ER compartment to migrate to the Golgi apparatus where it is cleaved by proteases. ${ }^{7}$ ATF 6 cytosolic fragment is a transcription factor responsible for the transcriptional induction of XBP1 as well as many ER chaperone-encoding genes. ${ }^{5}$ However, when cells are unable to recover from ER stress, apoptosis occurs.

The ER is an essential organelle for viral replication and maturation; in the course of productive infection, a large number of viral proteins are synthesized in infected cells, where unfolded or misfolded proteins activate the ER stress response. It is therefore not surprising that viruses have evolved various mechanisms to counteract these responses that limit or inhibit viral replication. For instance, $\mathrm{BiP}$ is induced in cells infected with respiratory syncytial virus, ${ }^{8}$ hanta viruses, ${ }^{9}$ hepatitis $\mathrm{C}$ virus $(\mathrm{HCV}),{ }^{10}$ bovine viral diarrhea virus (BVDV), ${ }^{11}$ Japanese encephalitis virus (JEV), ${ }^{12}$ dengue ${ }^{13}$ and enterovirus $71 .^{14}$ The induction of PERK has also been reported in infection with herpes simplex virus, ${ }^{15}$ cytomegalovirus (CMV) ${ }^{16}$ and BVDV. ${ }^{11}$ The IRE1 pathway has been shown to be activated in cells infected with JEV, dengue ${ }^{17}$ and severe acute respiratory syndrome virus, ${ }^{18}$ whereas the ATF6 pathway is triggered upon HCV infection ${ }^{19}$ and both IRE1 and ATF6 arms are induced during Rotavirus infection. ${ }^{20}$ In addition, the activation of all three pathways of the UPR has been reported in infection with West Nile virus ${ }^{21}$ and dengue. ${ }^{22}$ Furthermore, ASFV encodes the DP71L protein homolog to GADD34, ${ }^{23}$ which inhibits the induction of ATF4 and its downstream target CHOP. ${ }^{24,25}$ However, the infection does not induce the phosphorylation of PERK or the upregulation of $\mathrm{BiP} .{ }^{24}$

Here, we found that ASFV induces caspases 3, 9 and 12 but not caspase 8 in infected cells, as it regulates the three arms of the UPR signaling cascade, thus inhibiting the pathways that hinder viral infection and activating those that are beneficial.

\section{Results}

Activation of caspases in ASFV-infected cells. Caspases are key factors in the regulation of apoptotic pathways. These proteins are induced by a variety of apoptotic triggers (stimuli). To identify the apoptotic pathways induced in ASFV-infected cells, we performed caspase activity assays at a range of times after infection (Figure 1). ASFV induced caspases 3 and 9 in Vero (African green monkey kidney) cells from $16 \mathrm{~h}$ postinfection (hpi) and increased this activation along infection, with higher levels at $48 \mathrm{hpi}$ and both curves showed a similar pattern (Figures 1a and b). However, caspase 8 activity was not detected in infected Vero cells (Figure 1c). This observation was further confirmed in the infected WSL-R macrophage cell line (Figure 1d); however, caspase 8 activity was found in the specific positive control in both cases.

We also tested the activity of caspase 12, an ER membrane-associated cysteine protease that is activated in response to ER stress. ${ }^{26}$ Caspase 12 was undetectable in mock-infected cells or at $6 \mathrm{hpi}$, but at $16 \mathrm{hpi}$ it was induced and increased threefold until $48 \mathrm{hpi}$ (Figure 1e). The subcellular localization of this caspase in ASFV-infected cells was studied by indirect immunofluorescence (Figure 1f). As a control of induced caspase 12, Vero cells were treated with the ER stress-inducing agent tunicamycin, which activates caspase 12 and translocates it into the nucleus. Caspase 12 was localized in the nucleus in infected cells and in cells incubated with tunicamycin, but not in mock-infected ones.

To study the impact of apoptosis on ASFV infection, we infected Vero cells treated with an inhibitor of caspase 3 activation (Ac-DEVD-CHO) and with a specific inhibitor of myosin II ATPase activity (Blebbistatin). Myosin II ATPase activity, together with the GTPase ROCK-I, is necessary for cell membrane fragmentation into multiple apoptotic bodies in the execution phase of apoptosis. Also, we analyzed the effect of Y-27632, a ROCK-I inhibitor that blocks membrane blebbing. We then compared final infective virus production at $48 \mathrm{hpi}$ when these drugs were added to the cells, either $2 \mathrm{~h}$ before infection, or at $6 \mathrm{hpi}$. Our results showed that neither blebbing nor caspase 3 inhibition modified total virus production (extracellular plus intracellular virus production; Figure 2a). Caspase 3 activation in early stages of the infection was required for virus exit, whereas the inhibition of membrane blebbing reduced the release of virus particles from the cell and this effect was enhanced when the inhibitor was added $6 \mathrm{hpi}$ (Figure $2 \mathrm{~b}$ ). In order to asses a possible role of caspase 12 in caspase 3 and 9 activation, we analyzed the effect of caspase 12 inhibition in infected cells using Z-ATDFMK. Addition of this inhibitor before infection did not affect percentages of ASFV-infected cells as detected by flow cytometry (fluorescence-activated cell sorter (FACS); Figure 3a). Also, this inhibitor did not cause a decrease in virus production nor in the exit of virus particles (Figure $3 b$ ). Moreover, our results showed that neither caspase 3 nor caspase 9 were inhibited by Z-ATD-FMK and only caspase 12 activation was specifically inhibited (Figure $3 c$ ).

ASFV infection leads to the induction of ER chaperones. The induction of caspase 12 after ASFV infection suggests that the virus triggers an ER stress response. This response is an autoregulatory program that upregulates a large number of genes, such as ER chaperones and ER-associated degradation (ERAD) components, which increase the folding capacity of the ER. ${ }^{26}$ In order to determine whether ASFV infection induces this response, we used western blot analysis to monitor the expression levels of the ER chaperones BiP, calreticulin, calnexin, ERp57 and PDI (protein disulfide isomerase) over the course of the infection. Calreticulin and calnexin were induced upon ASFV infection at $16 \mathrm{hpi}$ (Figure 4a), while only a modest increase in PDI levels was found at $48 \mathrm{hpi}$. Interestingly, ASFV did not induce the expression of ERp57 or BiP. Chaperone upregulation was coincident with the expression of viral protein p30. Actin levels remained constant during the course of the infection, indicating that chaperone induction was not caused by a global upregulation of protein translation. We also analyzed the expression of BiP by inmunofluorescence. Cells treated with tunicamycin showed a typical ER pattern for BiP, while BiP expression was undetectable in non-treated/non-infected controls and infected cells (Figure 4c). This could be probably due to the low levels of $\mathrm{BiP}$ to be detected by the antibody.

Calnexin is an ER resident molecular chaperone that has a key role in the correct folding of membrane proteins. It has 

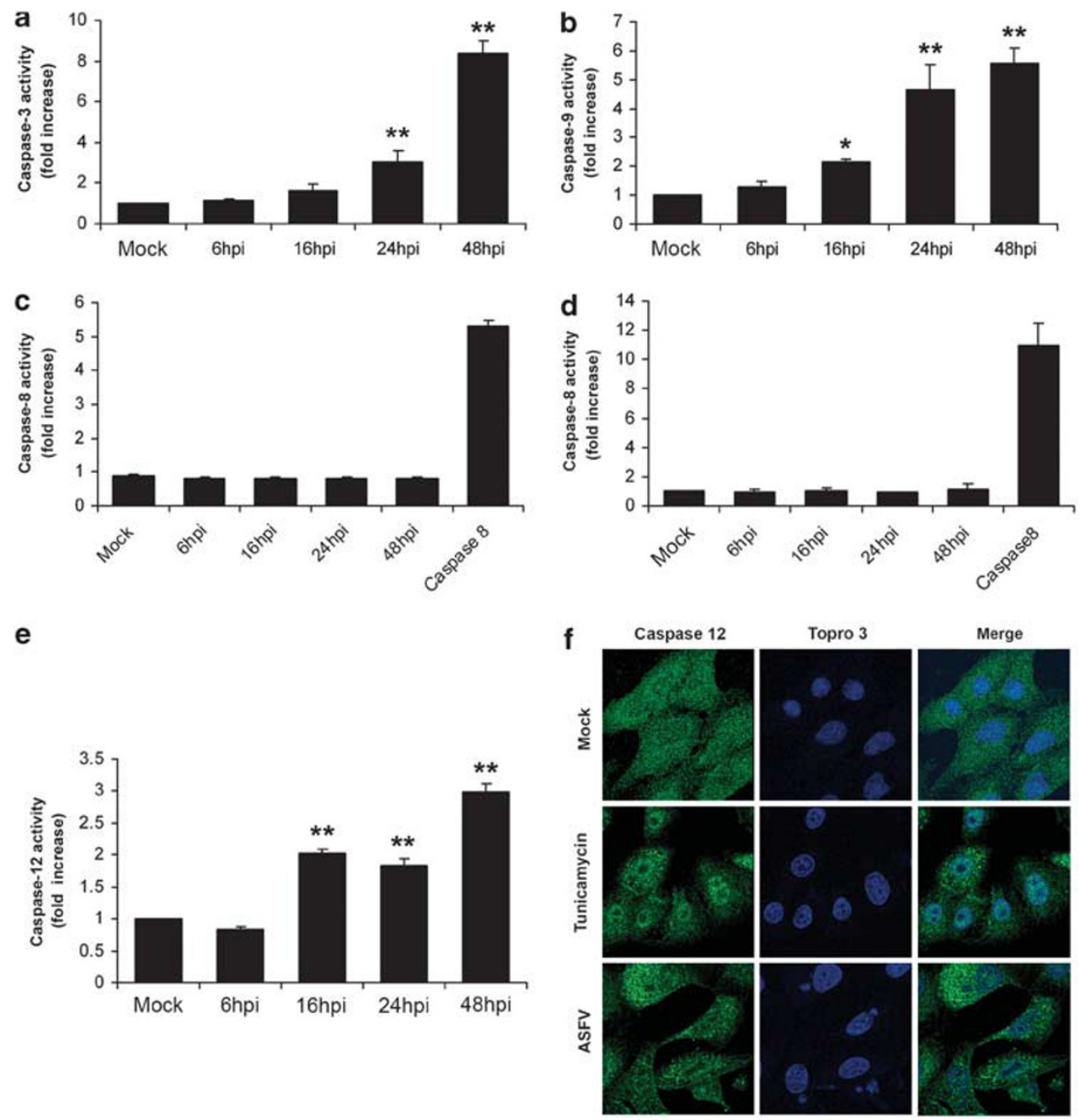

Figure 1 Caspase activities in ASFV-infected cells. Caspase activities were measured in virus-infected cell lysates using specific activity assays at the indicated time points after infection. Data were normalized to the corresponding values for mock-infected cells for each caspase. (a and $\mathbf{b})$ Caspase 3 and 9 activity of virus-infected Vero cells. (c and d) Caspase 8 activity of ASFV-infected Vero and WSL-R macrophage cell lines, respectively. (e) Caspase 12 activity of ASFV-infected Vero cells. (f) Representative confocal micrographs of nuclear localization of caspase 12 in ASFV-infected Vero cells. Cells treated with tunicamycin were used as controls. Statistical significance is indicated by asterisks $\left({ }^{\star} P<0.05 ;{ }^{*} P<0.01\right)$

been proposed that calnexin acts as a scaffold for caspase 8-dependent cleavage of the ER transmembrane protein Bap31 and thus for the generation of the pro-apoptotic p20 under ER stress. ${ }^{27}$ The Bap31 p20 fragment directs proapoptotic crosstalk between the ER and mitochondria. ${ }^{28}$ To study whether overexpression of calnexin during the infection is involved in the cleavage of Bap31, we monitored p20 by western blot. Bap31 remained intact during the infection, thereby indicating the absence of pro-apoptotic signals between the ER and mitochondria (Figure 4a).

ASFV infection does not result in an attenuation of protein translation. We studied the expression of UPR markers in virus-infected Vero cells. The activation of the PERK pathway results in the phosphorylation of the elF2 $\alpha$ subunit, leading to translation attenuation. ${ }^{29}$ PERK also activates the expression of ATF4, a transcription factor, thus leading to the upregulation of the pro-apoptotic genes $\mathrm{CHOP}$ and GADD34. ${ }^{30}$ GADD34 association with the phosphatase
PP1 produces the dephosphorylation of elF2 $\alpha{ }^{5}$ Tunicamycin treatment caused an increase in CHOP expression in Vero cells; however, no induction of $\mathrm{CHOP}$ was observed in ASFV-infected cells by western blot (Figure 5a). ATF4 expression was detected only in infected cells at $48 \mathrm{hpi}$. GADD34 protein showed an increase in expression from $16 \mathrm{hpi}$. Taken together, these results indicate that ASFV inhibits CHOP expression and leads to increased elF2 $\alpha$ dephosphorylation in order to restore protein translation.

ASFV infection does not induce XBP1 splicing in Vero cells. In response to the accumulation of unfolded proteins in the ER, IRE1 is activated by its oligomerization in the membrane, which causes the splicing of the mRNA encoding XBP1 transcription factor. The XBP1 protein encoded by the spliced mRNA is more stable than the unspliced form and is a potent transcription factor of the basic-leucine zipper (bZIP) family and caspase 12 inhibition flow cytometry (FACS ERAD) PDI elF2 $\alpha$ basic-leucine zipper (bZIP) one of the key 


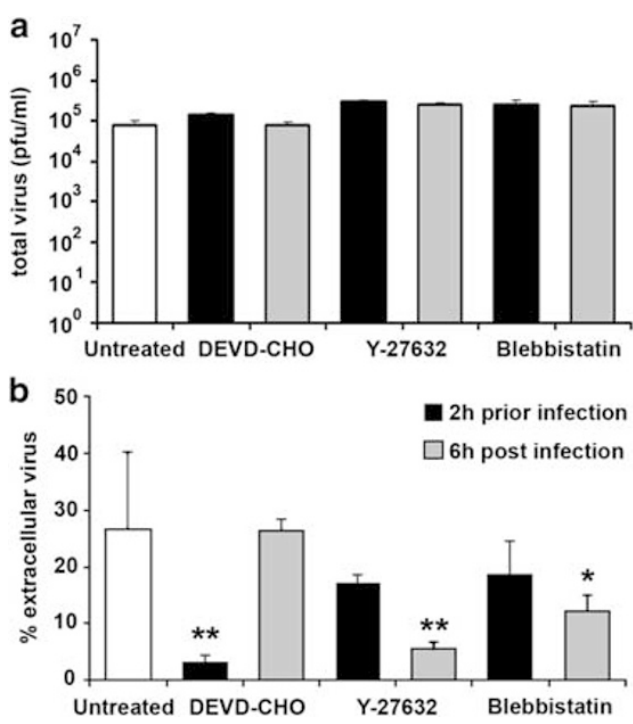

Figure 2 Caspase 3 activation and membrane blebbing enhance ASFV spread. Vero cells treated with DEVD-CHO, Y-27632 or Blebbistatin were infected with ASFV. Drugs were added $2 \mathrm{~h}$ before infection or $6 \mathrm{~h}$ after, as indicated. At $48 \mathrm{hpi}$, cells and media were harvested and titrated by plaque assay. Total (a) or extracellular virus titers (b) are shown. Error bars indicate S.D. from three independent experiments. Statistically significant differences are indicated by asterisks $\left({ }^{*} P<0.05\right)\left({ }^{* *} P<0.01\right)$

regulators of ER-folding capacity. ${ }^{31}$ Using RT-PCR and specific primers to distinguish between the unspliced (inactive) and spliced (active) forms of the XBP1 transcript, we detected XBP1 splicing in Vero cells treated with the UPR inducer tunicamycin but not in ASFV-infected cells at a range of post-infection times (Figure $5 \mathrm{c}$ ). Therefore, it appears that the infection did not increase levels of active XBP1 mRNA. XBP1 mRNA is spliced by IRE1 but is induced by ATF6 activation. ${ }^{6}$ The level of endogenous XBP1 mRNA was measured in infected cells using qRT-PCR analysis of RNAs from ASFV-infected samples. We found an increase in the level of XBP1 transcript in cells treated with tunicamycin or dithiothreitol (DTT) compared with the level of mRNA obtained in mock-infected cells. In contrasts, ASFV results in a gradual decrease in the level of XBP1 mRNA over the course of infection (Figure $5 \mathrm{~d}$ ) indicating the downregulation of this mRNA in ASFV-infected cells.

ASFV infection activates the ATF6 pathway. In response to ER stress, ATF6 exits the ER to the Golgi apparatus, where it is processed by proteases to its active form, which in turn translocates to the nucleus. In order to monitor ATF6 activation by fluorescence microscopy, we used an EGFP (enhanced green fluorescent protein)-ATF6 fusion protein (pCMV short-EGFP-ATF6). ${ }^{32}$ This protein was under the control of a shortened CMV promoter, which has a deletion of $430 \mathrm{bp}$ from the $5^{\prime}$ side in order to prevent overexpression, which would modify the localization of the fusion protein itself. The short promoter shows considerably less activity than the full promoter and GFP-ATF6 expressed under the short CMV promoter localizes exclusively to the ER and translocates to the nucleus, in a similar way to endogenous ATF6. ${ }^{32}$ Cells transfected with the pCMV short-EGFP-ATF6 plasmid presented a typical ER pattern that relocated to the Golgi apparatus (Figure 6b) or to the nucleus (Figure 6c) in response to tunicamycin treatment. However, this characteristic pattern was modified in infected cells. Cells transfected with this construct and infected with ASFV showed GFPATF6 localization either in discrete perinuclear cytoplasmic areas corresponding to viral factories, as indicated by their co-localization with DNA stain (Figures $6 \mathrm{~d}$ and e), or in the nucleus of infected cells (Figure 6f). This finding indicates that ASFV activates the ATF6 pathway of the UPR. We next studied the effect of a serine protease inhibitor that inhibits ER stress-induced proteolysis of ATF6, AEBSF ${ }^{33}$ on ASFV infection. Addition of this inhibitor before infection affected the percentages of ASFV-infected cells in a dose-dependent manner as detected by FACS (Figure 7a). AEBSF also induced a strong inhibition of viral production resulting in $100 \%$ reduction of viral titer at $300 \mu \mathrm{M}$ concentrations of AEBSF (data not shown). We analyzed caspase activation under AEBSF to find that this agent abrogated caspase 3, 9 and 12 activation in infected cells. Inhibition of caspases was dependent of AEBSF effect on infection, given that this serine protease inhibitor did not prevent activation of caspase 3 induced by staurosporine (Figure 7b).

\section{Discussion}

Apoptosis is a genetically controlled cell death mechanism involved in the regulation of tissue homeostasis. We analyzed the mechanism by which ASFV induces apoptosis in infected cells and observed that caspases 3 and 9 are activated with similar kinetics as of $16 \mathrm{hpi}$. However, ASFV-induced apoptosis was independent of caspase 8 activation, a process involved in the extrinsic apoptotic pathways. These results indicated that the virus induces apoptosis through the mitochondrial pathway rather than the death receptormediated pathway. ASFV triggers apoptosis in an early stage of the infection process. ${ }^{34}$ Nevertheless, extensive apoptosis of infected cells occurs only at late post-infection times. Programmed cell death itself might be relevant for the release of virus particles into apoptotic bodies, which would provide a mechanism to facilitate virus spread and evasion of the immune system. Our results show that caspase 3 activation in early stages of the infection, was relevant for virus exit and that membrane blebbing contributes to virus dissemination, given the fact that the inhibition of the effector caspase 3 reduced the release of virus particles from the cell and this effect was enhanced when the inhibitor was added after $6 \mathrm{hpi}$. Thus, ASFV takes advantage of an early induction of apoptosis, through caspase 3 activity, while the final execution of programmed cell death in apoptotic blebs contribute to ASFV invasion of host cells and evasion of the immune surveillance. In addition to its critical role in apoptosis, caspase 3 participates in other cellular processes. ${ }^{35}$ This could also be the case of ASFV infection, where activation of caspase 3 may be required during early stages of infection to ensure subsequent virus exit from the cell. Another example is the influenza virus infection, in which caspase 3 inhibitors prevent the formation of progeny influenza $A$ virus particles. ${ }^{36}$

We have also shown that ASFV infection in Vero cells induces caspase 12, although this induction is not required for 

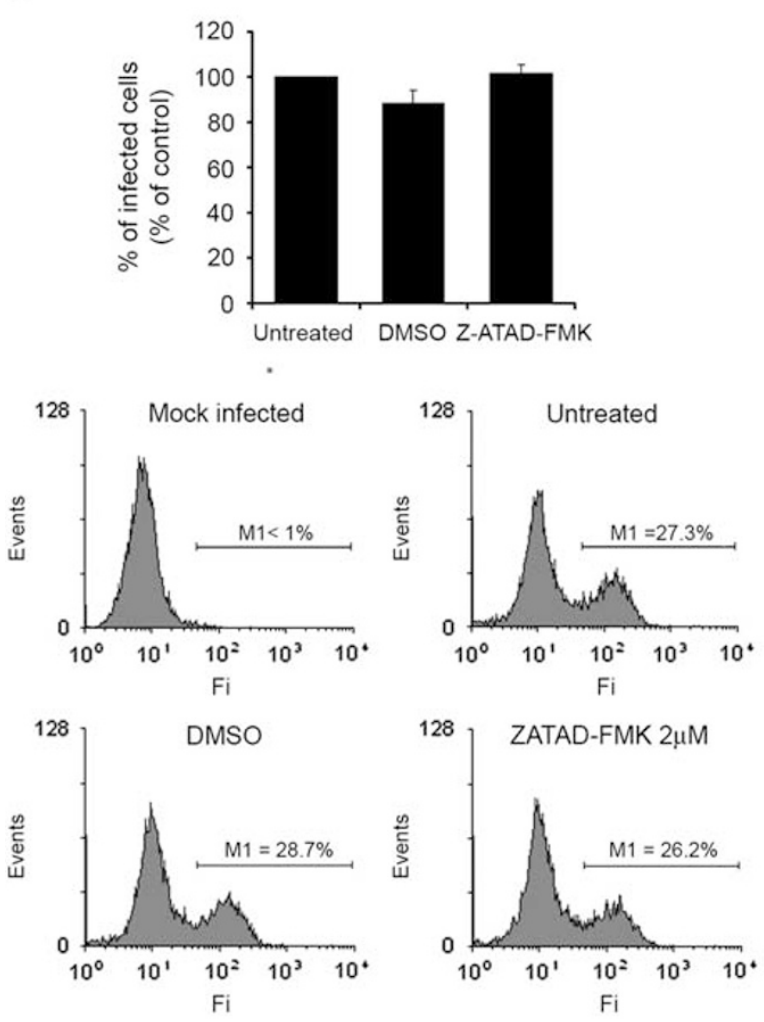
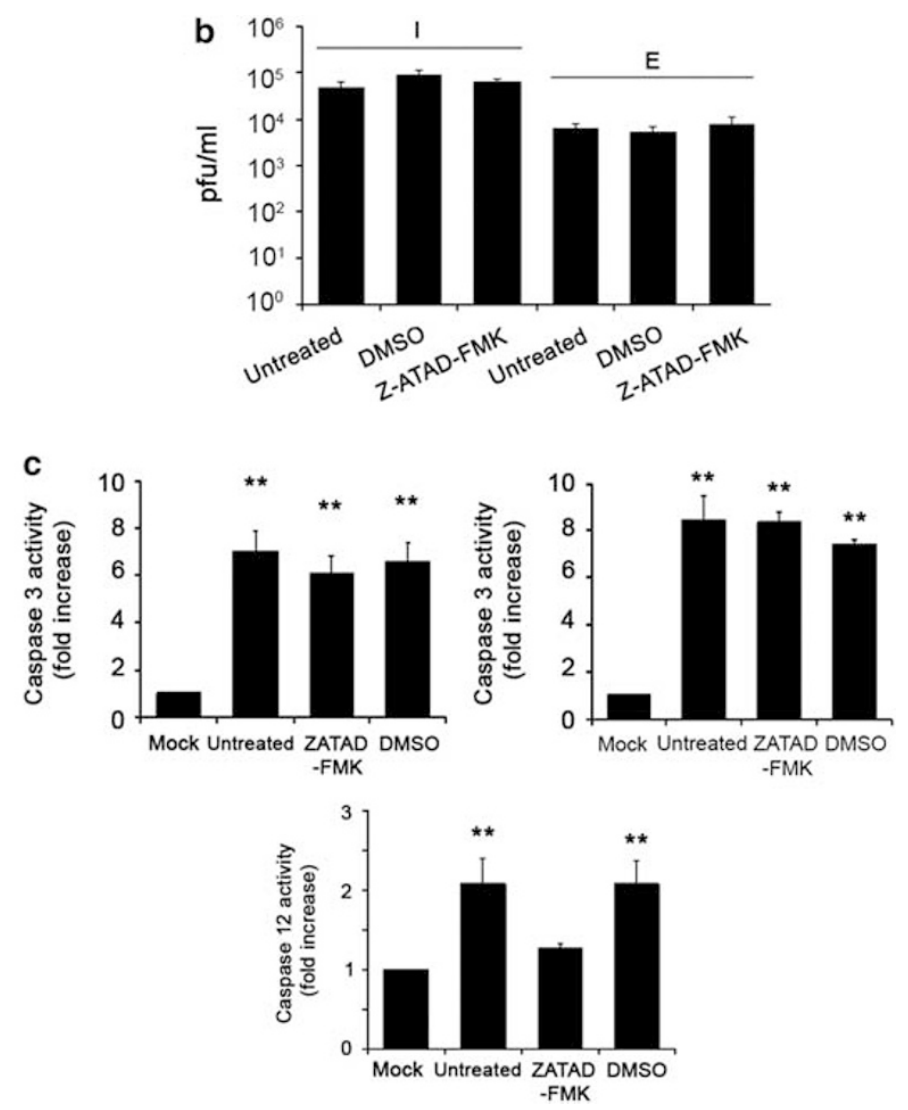

Figure 3 ASFV infection is independent of caspase 12 activation. Vero cells were pretreated with Z-ATAD-FMK and then infected with ASFV. (a) Infected cells were detected by FACS and percentages normalized to values in untreated cells. Representative FACS profiles are shown in the graphs below. Infected cells were gated in M1 and expressed as a percentage of total cells analyzed. (b) Cells and media were harvested at $48 \mathrm{hpi}$ and titrated by plaque assay. Intracellular (I) or extracellular (E) virus titers in $\mathrm{pfu} / \mathrm{ml}$ are shown. (c) Caspase activities were analyzed at $24 \mathrm{hpi}$ using specific activity assays. Data were normalized to control values. Error bars indicate S.D. from three independent experiments and statistically significant differences are indicated by asterisks $\left.{ }^{* \star} P<0.01\right)$

viral production or for caspases 3 and 9 activation. Caspase 12 is a characteristic ER stress response caspase. Virus assembly begins with the modification of ER membranes, which are subsequently recruited to the viral factories and transformed into viral precursor membranes. ${ }^{37}$ Moreover, a large amount of viral proteins are synthesized and accumulated in infected cells, thus overloading the ER-folding capacity. This overload can lead to the activation of the ER stress response of the host cell. Activated caspase 12, a key marker of ER stress-mediated apoptosis, may potentially interact with another pro-apoptotic protein, Bap31, resulting in a feedback mechanism involving the release of cytochrome $c$ elicited through the mitochondrial pathway. ${ }^{38}$ However, Bap31 was not activated by the fragmentation of p20 in ASFV-infected cells. This observation indicates the absence of direct pro-apoptotic signals between the ER and mitochondria. Caspase 12 was triggered in ASFV-infected cells but this activation does not necessarily result in apoptosis, because the virus has the capacity to block ER stress at a later step. In general, ER stress starts with the transcriptional induction of ER-localized chaperones as a survival signal. Molecular chaperones are key components of the ER machinery; their main function consists of ensuring the quality of the ER by maintaining newly synthesized proteins in a proper state for folding, and marking misfolded proteins for degradation. Two folding systems have been proposed to contribute to this quality control. One of these is composed of grp78 (BiP), grp94 and PDI. The second system, known as the 'calnexincalreticulin cycle', includes calnexin, calreticulin and ERp57. ${ }^{39}$ Chaperone expression analysis of ASFV-infected cells revealed a marked increase in calnexin and calreticulin expression from $16 \mathrm{hpi}$, and to a lesser extent, of PDI at $48 \mathrm{hpi}$. However, ASFV did not induce the expression of ERp57, which, together with calnexin and calreticulin, assists disulfide bond formation in glycoprotein folding. Surprisingly, ASFV did not elicit the expression of grp78 (BiP), thereby indicating that the virus contains the ER stress at this level.

To determine whether ER stress is involved in ASFVinduced cellular responses, we analyzed several ER stressrelated proteins, including $\mathrm{CHOP}, \mathrm{GADD} 34, \mathrm{XBP} 1, \mathrm{ATF} 6$ and ATF4. Previous results showed that viral infection in Vero cells inhibits the induction of the ATF4-CHOP signaling arm of the UPR. $^{24,25}$ However, we observed expression of ATF4 from $48 \mathrm{hpi}$ when virus is thought to promote programmed cell death in order to spread progeny. The inhibition of this UPR branch results in an attenuation of protein translation. To avoid this, ASFV encodes the DP71L gene, a GADD34 homolog that interacts with the catalytic subunit of PP1, and activates its phosphatase activity ${ }^{40}$ and that also promotes the expression of cellular GADD34, as shown here. 

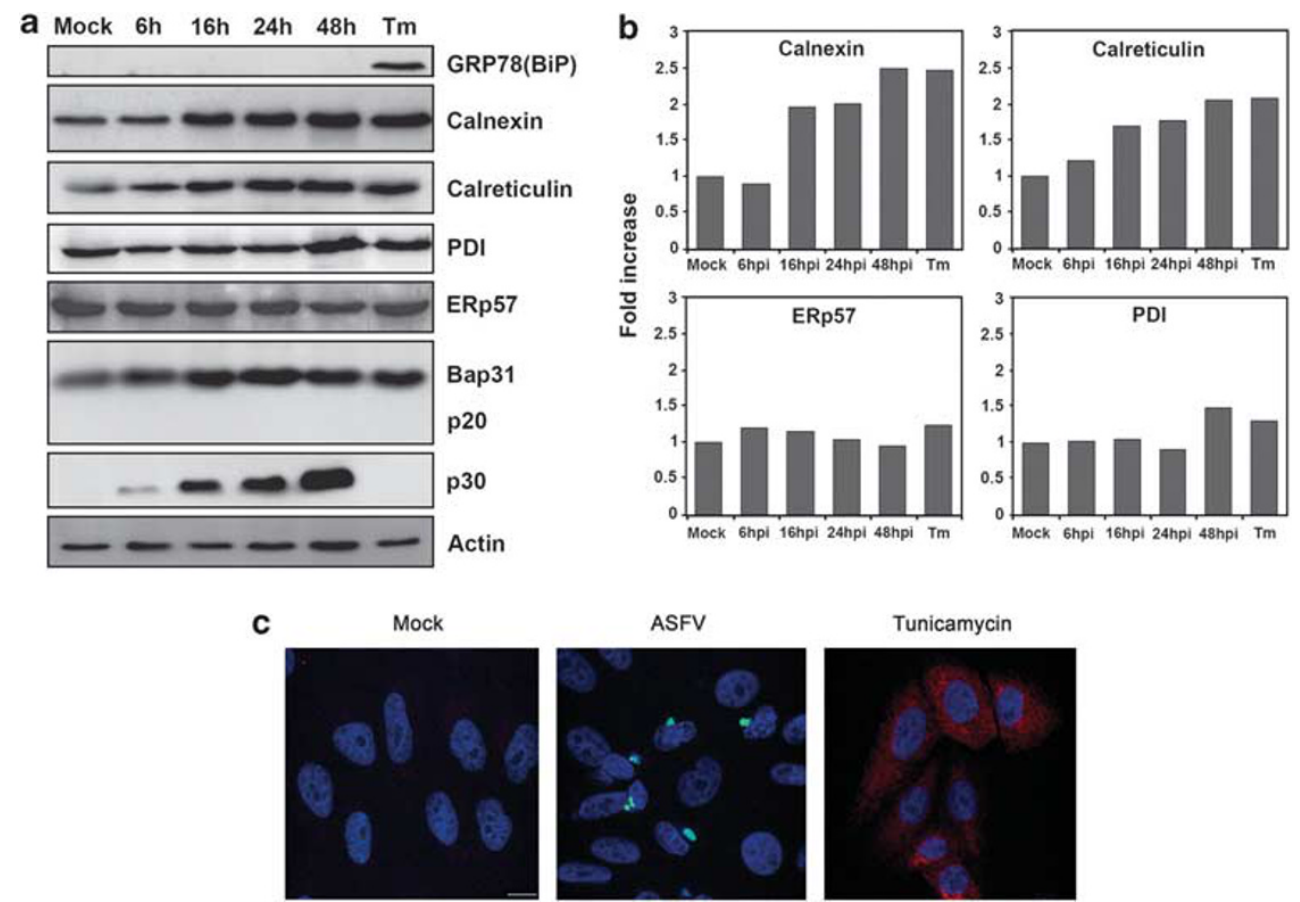

Figure 4 ASFV induces the expression of ER chaperones but not the expression of ERp57 or fragmentation of Bap31. (a) Western blot analysis of the ER chaperones in Vero cells infected with ASFV or treated with tunicamycin $(\mathrm{Tm})$. Cell lysates were harvested at the indicated times after infection and then, analyzed. Actin was used as protein load control and viral infection was followed by p30 viral protein expression. (b) Quantification of the bands by densitometry corrected to actin data and normalized to control values. (c) Representative confocal micrographs of Vero cells grown on glass slides were treated with tunicamycin or infected with B54GFP-2 for $16 \mathrm{hpi}$. Cells were fixed, stained with anti-BiP plus Alexa Fluor 594-conjugated secondary antibody and incubated with Topro-3 for DNA staining and then analyzed by confocal fluorescence microscopy. Bar $10 \mu \mathrm{M}$
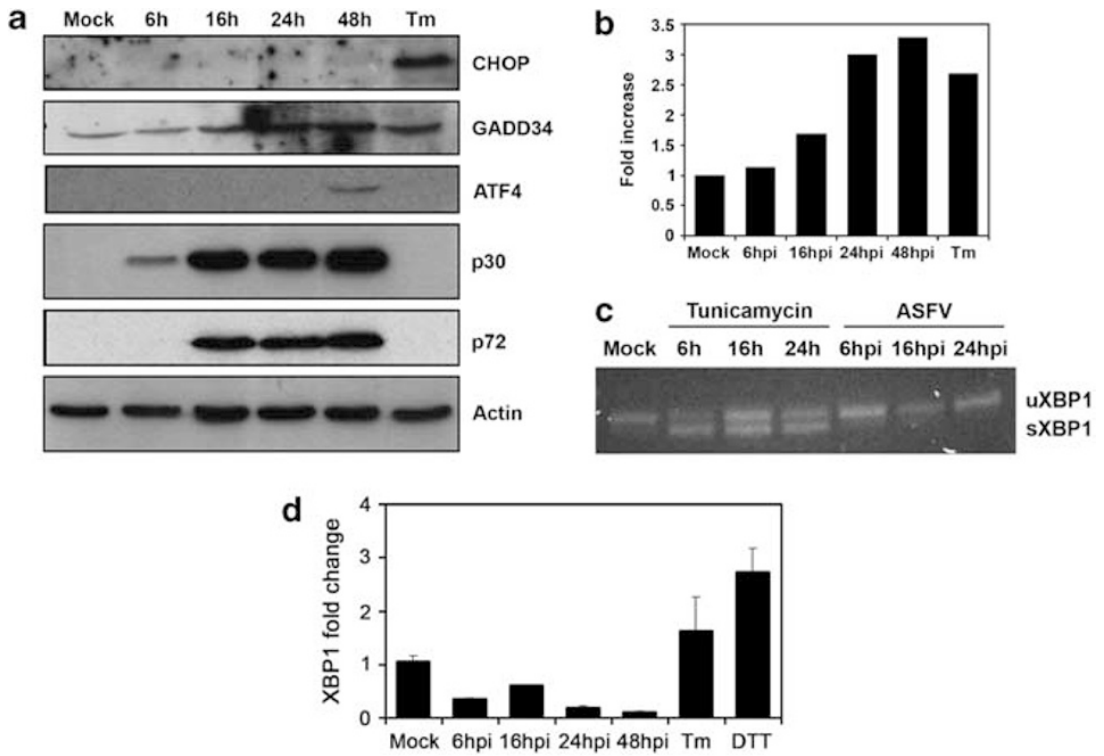

Figure 5 ASFV regulates UPR signaling. (a) Western blot analysis of CHOP, GADD34, ATF4, p30 and p72 viral proteins in Vero cells infected with ASFV or treated with tunicamycin, lysated at the indicated times after infection with actin as protein load control. (b) Quantification of the bands corresponding to GADD34 by densitometry was corrected to actin data and normalized to control values (c) Unspliced (UXBP1) and spliced (sXBP1) bands were RT-PCR-amplified using specific primer pairs. Cells treated with tunicamycin were used as a positive control for the induction of spliced XBP1. (d) XBP1 mRNA levels quantified with real-time RT-PCR. Total RNA was isolated from ASFV-infected Vero cells at a range of times post-infection. The XBP1 message was normalized to the 18S ribosomal message and $x$-fold changes were calculated as described in Materials and Methods. DTT and Tm-treated cells were used as control. Data are means \pm S.D. from three independent experiments 
GFP-ATF6

a

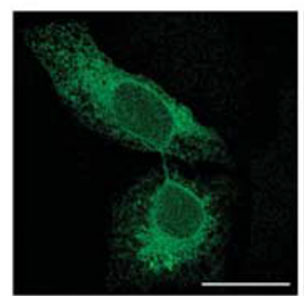

b

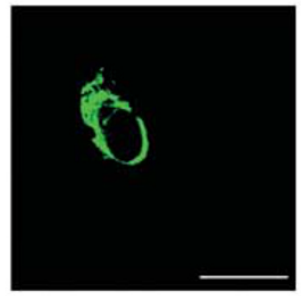

c

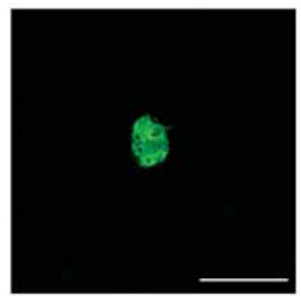

d

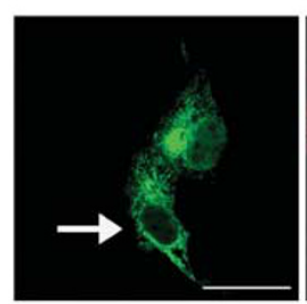

e
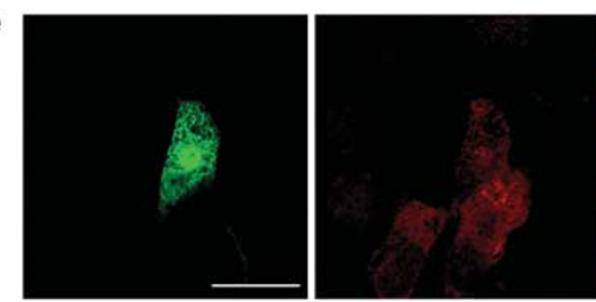

f
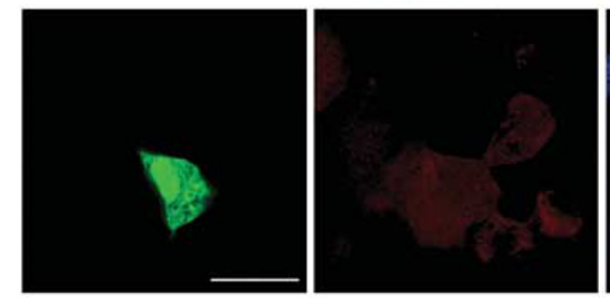

g

\begin{tabular}{r|cccc}
$\%$ & ER & Golgi & Nucleus & Viral factory \\
\hline Mock & 100 & - & - & - \\
Tunicamycin & 7.1 & 39.3 & 53.6 & - \\
Infected cells & 21 & 5.3 & 44.7 & 29
\end{tabular}

Topro3
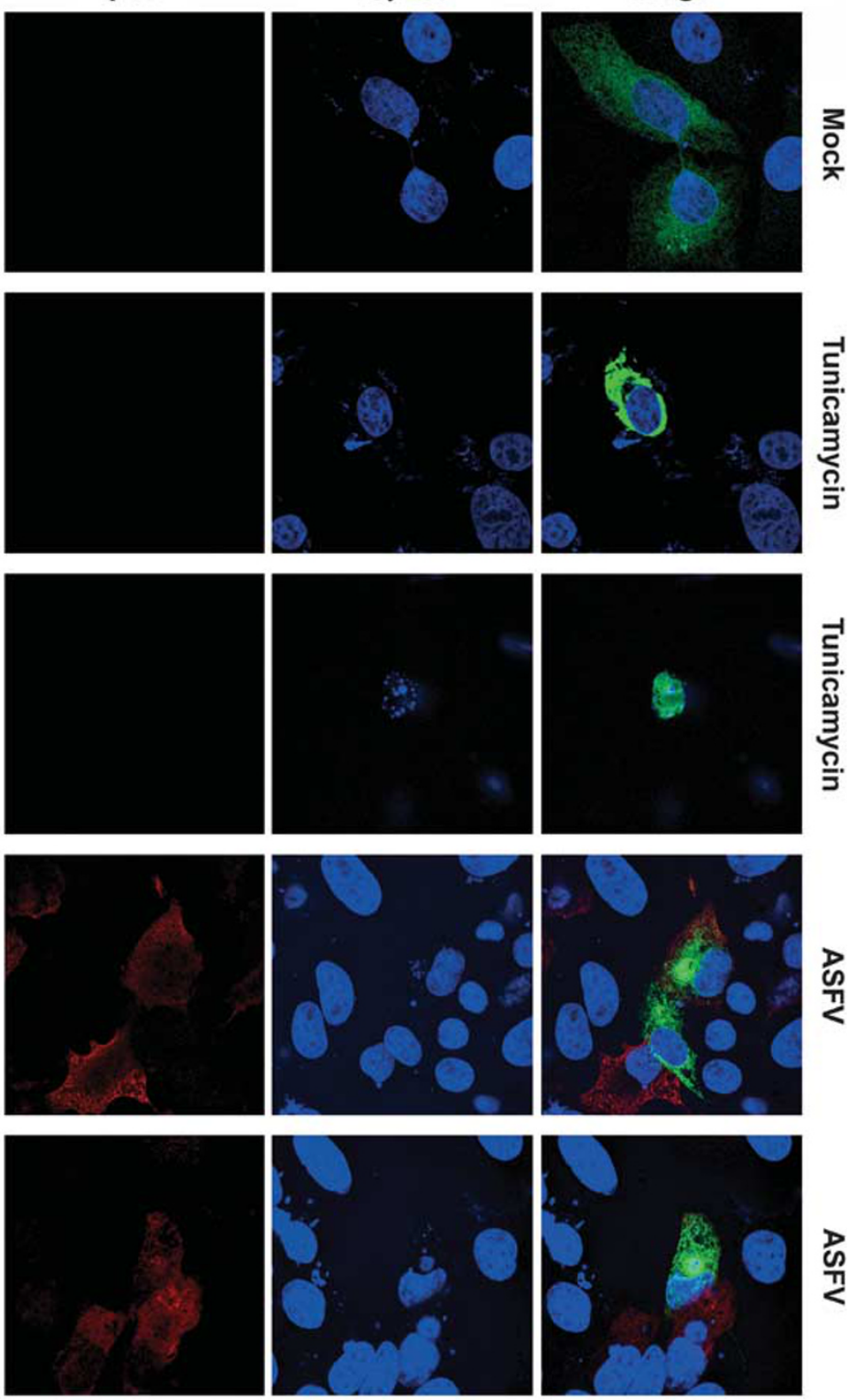

${ }_{0}^{\pi}$

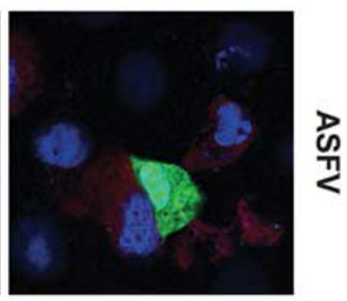

\section{变}

Figure 6 ASFV infection activates the ATF6 pathway. (a) Representative confocal micrographs of Vero cells transfected with the GFP-ATF6 plasmid. After $24 \mathrm{~h}$, untreated cells showed a typical ER pattern, while in cells treated with tunicamicyn, GFP-ATF6 relocated to the Golgi (b) and to the nucleus (c). (d-f) Representative confocal micrographs of ASFVinfected transfected cells. ATF6 translocated to the viral factories and to the nucleus. (d, arrow) A neighboring uninfected cell is shown for comparison. Bar $25 \mu \mathrm{m}$. (g) Percentages of GFP-ATF6 subcellular localization events in infected and tunicamycin treated cells in triplicate experiments calculated in a total of $5 \times 10^{4}$ cells/well 
a
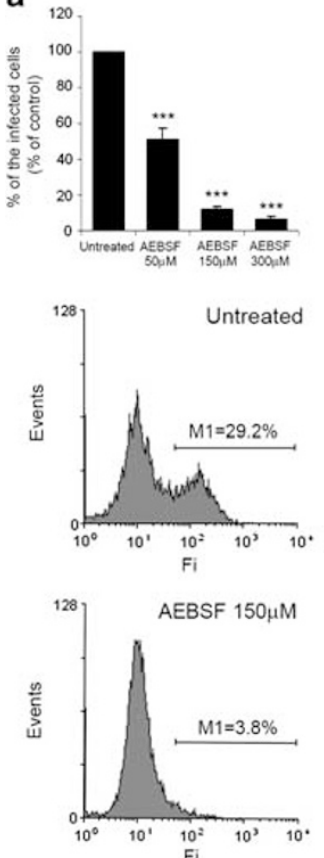
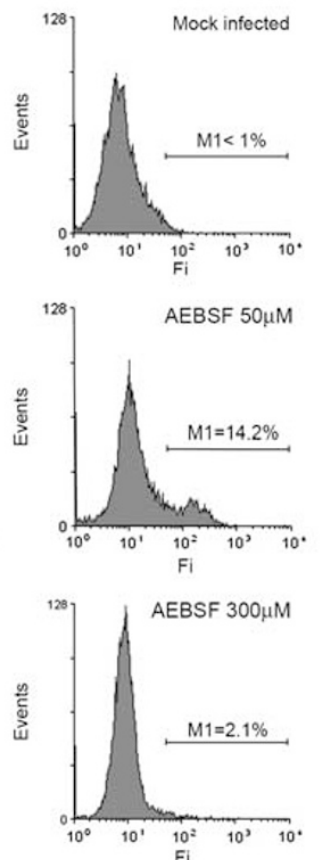

b
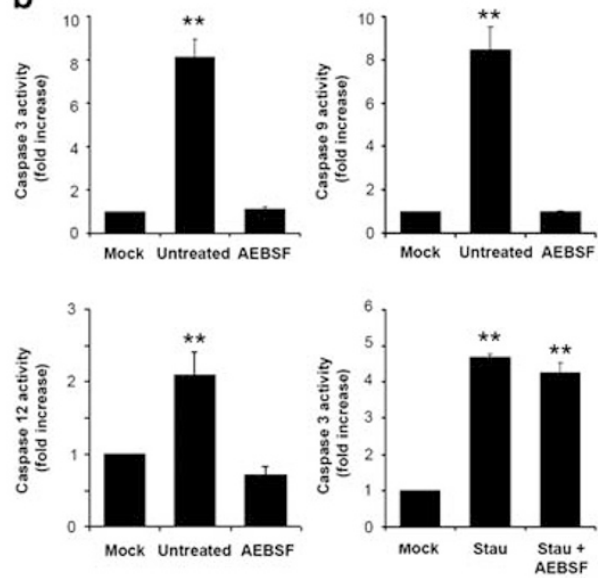

Figure 7 ASFV infection is dependent on ATF6 activation. (a) ASFV-infected cell percentages in Vero cells pretreated with AEBSF at the indicated concentrations and then infected. Infected cells were detected by FACS and data normalized to infection rates in untreated cells. Representative FACS profiles obtained during the analysis are shown. Infected cells were gated in M1 and expressed as a percentage of total cell analyzed. (b) Caspase activities at $24 \mathrm{hpi}$ in Vero cells pretreated with $300 \mu \mathrm{M}$ AEBSF and then infected. Data were normalized to mock-infected control values. Cells treated with Staurosporine (Stau) were used as control. Asterisks denote statistically significant differences $\left({ }^{\star *} P<0.01,{ }^{* * *} P<0.001\right)$

IRE1-XBP1 is also critical for the UPR, as it stimulates the expression of proteins involved in ER stress-induced protein degradation. Nevertheless, no splicing of XBP1 was detected during ASFV infection. The absence of splicing may favor viral infection as this process would inhibit viral protein degradation induced by ER stress.

The activation of the ATF6 branch and its transcriptional activation of chaperone-encoding genes might benefit the virus by assisting the folding of accumulated proteins and preventing protein aggregation. We demonstrated ATF6 translocation to the nucleus. Activated ATF6 exits the ER to the Golgi apparatus, where it is processed by proteases to its active form, which in turn translocates to the nucleus. ${ }^{7}$ We used a short promoter that has considerably lower activity than the full promoter, and GFP-ATF6 expressed under the short CMV promoter localizes exclusively to the ER and translocates to the nucleus in a similar manner to endogenous ATF6. ${ }^{32}$ The analysis of ATF6 distribution by confocal microscopy in Vero cells infected with ASFV revealed that this protein was translocated into the nucleus. ATF6 was also located in discrete perinuclear cytoplasmic areas that corresponded to viral factories, as indicated by the colocalization of ATF6 with DNA-containing cytoplasmic foci. Furthermore, ASFV viral replication was blocked by inhibition of the ATF6 translocation to the nucleus by AEBSF. To analyze the transcriptional activity of ATF6 in ASFV infection we examined the expression of XBP1 in ASFV-infected cells by qRT-PCR. Surprisingly, ASFV-infected samples showed a decrease in the induction of XBP1 compared with uninfected samples. Activation of ATF6 was necessary for ASFV replication; however, the virus prevented the transcriptional induction of some ATF6-target genes, such as XBP1 and BiP, but not calnexin or calreticulin. These studies have focused interest in ATF6 pathway in this viral infection and have paved the way for further characterization of ASFV viral gene regulation of these molecules in the UPR.

In conclusion, apoptosis induction, caspase activation and later modulation of the UPR might be advantageous for ASFV as these processes block the effects that are detrimental to the infection, while maintaining those that are beneficial.

\section{Materials and Methods}

Cell culture, viruses and plasmids. Vero cell lines were obtained from the American Type Culture Collection (ATCC, Richmond, VA, USA) and cultured at $37^{\circ} \mathrm{C}$ in Dulbecco's modified Eagle's medium supplemented with $5 \%$ fetal bovine serum (FBS), $100 \mathrm{IU} / \mathrm{ml}$ penicillin, $100 \mu \mathrm{g} / \mathrm{ml}$ streptomycin and $2 \mathrm{mM}$ L-glutamine. WSL-R line 379 , a macrophage cell line of wild swine origin that was kindly provided by Günther Kiel (Friedrich-Loeffler Institut, Greifswald, Germany), was grown in Iscove's medium (Gibco-BRL, Grand Island, NY, USA) plus F-12 nutrient mixture (Gibco) plus $10 \%$ FBS. Where indicated, cells were treated with $1 \mu \mathrm{g} / \mathrm{ml}$ Staurosporine (Sigma-Aldrich, Steinheim, Germany), or with $10 \mu \mathrm{g} / \mathrm{ml}$ tunicamycin (Sigma-Aldrich) or $2.5 \mathrm{mM}$ DTT (Sigma-Aldrich) as a positive control of ER stress.

The Vero-adapted ASFV isolate BA71V was used. For flow cytometry (FACS) analyses we used an infectious recombinant ASFV, B54GFP-2, which expresses and incorporates into the virus particle a chimera of the p54 envelope protein fused to the EGFP. ${ }^{41}$ Preparation of viral stocks, titrations and infection experiments were carried out in Vero cells as previously described. ${ }^{42}$

The plasmid pCMVshort-EGFP-ATF6 $\alpha$ was kindly provided by Dr. Mori (Kyoto University, Japan) ${ }^{32}$ and was transfected into Vero cells using Fugene 6 (Roche Biochemicals, Mannheim, Germany), following the manufacturer's protocols. 
Western blotting. Cells seeded in 24-well plates were harvested in Laemml sample buffer, boiled for $5 \mathrm{~min}$ at $95^{\circ} \mathrm{C}$, resolved by SDS-PAGE and transferred to a nitrocellulose membrane (Bio-Rad, Hercules, CA, USA). The non-specific antibody binding sites were blocked with skimmed milk in phosphate buffered saline (PBS) and then incubated with the specific primary and the HRP (Horseradish peroxidase)-conjugated secondary antibody.

Bands obtained after development with $\mathrm{ECL}$ reagent (GE Healthcare, Piscataway, NJ, USA) were detected using a Chemidoc XRS imaging system (Bio-Rad).

Antibodies used for western blotting included the following: rabbit polyclonal antibody to calreticulin (ab2907 abcam, Cambridge, UK) at a dilution of 1:3000; rabbit polyclonal antibody to calnexin (StressGen, San Diego, CA, USA) 1:3000; rabbit polyclonal to Bap31 (ab37120 abcam) 1:3000; rabbit polyclonal antibody to ERp57 (ab10287 abcam) 1:1000; goat polyclonal to GADD34 (ab9869 abcam) 1:1000; rabbit polyclonal antibody GADD153/CHOP (F-168) (Santa Cruz Biotechnology, Santa Cruz, CA, USA) 1:200; rabbit polyclonal antibody to GRP78 (BiP) (Santa Cruz Biotechnology) 1:200; rabbit polyclonal antibody to ATF4 (ab85049) 1:500 mouse monoclonal antibody to PDI (ab2792 abcam) 1:1000; anti$\beta$-actin (Sigma, St Louis, MI, USA) 1: 1500; anti-ASFV p30 monoclonal antibody 1:1000; and anti-p72 monoclonal antibody clone 18BG3 (Ingenasa, Madrid, Spain) $1: 5000$. The following secondary antibodies were used: goat anti-rabbit lgG HPR conjugate (Bio-Rad) and sheep anti-mouse IgG HPR conjugate (GE Healthcare), observing the manufacturer's instructions. Band densitometry was performed with TINA image analysis software and data were normalized to control values.

Caspase activity assays. Using fluorimetric caspase 3,8 and 9 assay kits (Sigma-Aldrich), the activity of caspases 3,8 and 9 , respectively, were measured at a range of times post-infection in Vero cells and in WSL-R cells infected with ASFV at a multiplicity of $1 \mathrm{pfu} / \mathrm{cell}$. Caspase 12 activity was determined using a fluorimetric caspase 12 assay kit (Biovision, Mountain View, CA, USA), following the manufacturer's instructions.

Effect of inhibitors on viral production. Monolayers of Vero cells were infected with ASFV at a multiplicity of infection of 1 pfu/cell and treated with Ac-DEVD-CHO ( $N$-acetyl-L-aspartyl-L- $\alpha$-glutamyl- $N$ - (2-carboxyl-1-formylethyl)-Lvalinamide) (Sigma-Aldrich), Blebbistatin (Sigma-Aldrich), Y-27632 (Calbiochem, Merck, Darmstadt, Germany), Z-ATAD-FMK (benzyoxycarbonyl-Ala-Thr-Aspfluoromethylketone) (Biovision) or AEBSF (4-(2-Aminoethyl)-Benzenesulfonyl Fluoride) (Sigma-Aldrich) at final concentrations of 25, 30, 10, 2 and $300 \mu \mathrm{M}$, respectively, for $2 \mathrm{~h}$ before the infection or $6 \mathrm{hpi}$. Infection was allowed to proceed for $48 \mathrm{~h}$ and then virus titers in cell lysates (intracellular virus) and culture medium (extracelullar virus) were titrated by plaque assay in triplicate samples on cells.

FACS analyses. Vero cells were pretreated with inhibitors at the indicated concentrations in growth medium for $1 \mathrm{~h}$ at $37^{\circ} \mathrm{C}$, followed by infection with ASFV B54GFP-2 recombinant virus at a moi of $1 \mathrm{pfu} / \mathrm{ml}$. After $24 \mathrm{hpi}$ cells were washed with PBS, harvested by trypsinization, and then washed with FACS buffer (PBS, $0.01 \%$ sodium azide, and $0.1 \%$ bovine serum albumin. In order to determine the percentage of infected cells per condition, 10000 cells/time point were scored and analyzed in a FACSCalibur flow cytometer (BD Sciences, Franklin Lakes, NJ, USA). Untreated control infected cultures yielded $25-30 \%$ of infected cells from the total cells examined. Infected cell percentages obtained after drug treatments were normalized to values found in control plates.

Immunofluorescence. Cells seeded on glass coverslips in a 24-well plate were infected with ASFV. At several time points after virus infection, the cells were fixed with $4 \%$ paraformaldehyde in PBS for $15 \mathrm{~min}$ at room temperature and permeabilized in $0.1 \%$ Triton X 100 in PBS for $15 \mathrm{~min}$ at room temperature. Cells were consecutively stained with primary and secondary antibodies and then incubated with Topro-3 (Invitrogen, Eugene, OR, USA) 1/1000 in PBS1X for DNA staining. After washing, coverslips were finally mounted on glass plates and cells were observed under a Leica TCS SPE confocal microscope (Leica-Microsystems, Wetzlar, Germany). The primary antibodies used were: rabbit polyclonal antibody to GRP78 (BiP) (Santa Cruz Biotechnology) 1:50, rabbit polyclonal antibody to caspase 12 (BioVision) diluted 1:25 and anti-ASFV p30 monoclonal antibody 1:100. Antirabbit IgG Alexa 488 and anti-mouse IgG Alexa 594-conjugated antibodies (Invitrogen) diluted to 1:200 were used as secondary antibodies respectively.
RNA preparation and RT-PCR analysis for XBP1. Total RNA from cultured cells was isolated with TRI-Reagent (Sigma-Aldrich) following the manufacturer's instructions. Reverse transcription reactions (RT-PCR) were carried out using the SuperScript One Step RT-PCR (Invitrogen), following the manufacturer's instructions. The primers used were: forward primer: $5^{\prime}$-CCTTGTAGTTGAGAAC CAGG-3' and reverse primer $5^{\prime}$-GGGGCTTGGTATATATGTGG-3'. Amplification conditions included an initial step of $45^{\circ} \mathrm{C}$ for $30 \mathrm{~s}$, followed by a denaturation step of $94^{\circ} \mathrm{C}$ for $2 \mathrm{~min}, 30 \mathrm{cycles}$ of $94^{\circ} \mathrm{C}$ for $30 \mathrm{~s}, 55^{\circ} \mathrm{C}$ for $30 \mathrm{~s}$ and $70^{\circ} \mathrm{C}$ for $30 \mathrm{~s}$. PCR products were resolved by electrophoresis in a $2 \%$ agarose gel and stained with SYBR Green.

For quantitative PCR, cDNA was synthesized using the QuantiTect Reverse Trancription kit (Qiagen, Hilden, Germany) following the manufacturer's instructions. After heat inactivation at $95^{\circ} \mathrm{C}, \mathrm{cDNA}$ levels were quantified by using quantitative PCR (qPCR). Amplification was performed in a Rotor-Gene 3000 (Corbett Life Sciences, Sydney, Australia) using a QuantiTect SYBR Green PCR kit (Qiagen) following the manufacturer's recommendations. Primer sets used for 18S and XBP1 are available from Qiagen (QuantiTect Primer Assay). Fold change was calculated using the $\Delta \Delta C T$ method of relative quantification with $18 \mathrm{~S} r R N A$ as the endogenous control for normalization. ${ }^{43}$ Melting curves were used to verify the specificities of products.

Data analysis. One-way analysis of variance was performed using INSTAT 3 for Windows. For multiple comparisons, Dunnet's correction was applied. Data are presented as means \pm S.D. Differences were considered statistically significant at a $P$-value of $<0.05$.

\section{Conflict of Interest}

The authors declare no conflict of interest.

Acknowledgements. We thank K Mori for the GFP-ATF6 construct. This work was supported by grants from the Spanish Ministry of Science and Innovation Program Consolider CSD 2006-00007, AGL2009-09209 and the Wellcome Trust Foundation WT075813.

1. Ramiro-lbanez F, Ortega A, Brun A, Escribano JM, Alonso C. Apoptosis: a mechanism of cell killing and lymphoid organ impairment during acute African swine fever virus infection. J Gen Virol 1996; 77(Pt 9): 2209-2219.

2. Brun A, Rivas C, Esteban M, Escribano JM, Alonso C. African swine fever virus gene A179L, a viral homologue of bcl-2, protects cells from programmed cell death. Virology 1996; 225: 227-230.

3. Thornberry NA, Lazebnik Y. Caspases: enemies within. Science 1998; 281: 1312-1316.

4. Rao RV, Hermel E, Castro-Obregon S, del Rio G, Ellerby LM, Ellerby HM et al. Coupling endoplasmic reticulum stress to the cell death program. Mechanism of caspase activation. J Biol Chem 2001; 276: 33869-33874.

5. Schroder M, Kaufman RJ. The mammalian unfolded protein response. Annu Rev Biochem 2005; 74: 739-789.

6. Yoshida H, Matsui T, Yamamoto A, Okada T, Mori K. XBP1 mRNA is induced by ATF6 and spliced by IRE1 in response to ER stress to produce a highly active transcription factor. Cell 2001; 107: 881-891.

7. Ye J, Rawson RB, Komuro R, Chen X, Dave UP, Prywes $R$ et al. ER stress induces cleavage of membrane-bound ATF6 by the same proteases that process SREBPs. Mol Cell 2000; 6: 1355-1364

8. Bitko V, Barik S. An endoplasmic reticulum-specific stress-activated caspase (caspase-12) is implicated in the apoptosis of A549 epithelial cells by respiratory syncytial virus. J Cell Biochem 2001; 80: 441-454

9. Li XD, Lankinen H, Putkuri N, Vapalahti O, Vaheri A. Tula hantavirus triggers pro-apoptotic signals of ER stress in Vero E6 cells. Virology 2005; 333: 180-189.

10. Liberman E, Fong YL, Selby MJ, Choo QL, Cousens L, Houghton M et al. Activation of the grp78 and grp94 promoters by hepatitis C virus E2 envelope protein. J Virol 1999; 73: 3718-3722.

11. Jordan R, Wang L, Graczyk TM, Block TM, Romano PR. Replication of a cytopathic strain of bovine viral diarrhea virus activates PERK and induces endoplasmic reticulum stressmediated apoptosis of MDBK cells. J Virol 2002; 76(19): 9588-9599.

12. Su HL, Liao CL, Lin YL. Japanese encephalitis virus infection initiates endoplasmic reticulum stress and an unfolded protein response. J Virol 2002; 76: 4162-4171.

13. Wati S, Soo ML, Zilm P, Li P, Paton AW, Burrell CJ et al. Dengue virus infection induces upregulation of GRP78, which acts to chaperone viral antigen production. J Virol 2009; 83: 12871-12880.

14. Jheng JR, Lau KS, Tang WF, Wu MS, Horng JT. Endoplasmic reticulum stress is induced and modulated by enterovirus 71. Cell Microbiol 2010; 12: 796-813. 
15. Cheng G, Feng Z, He B. Herpes simplex virus 1 infection activates the endoplasmic reticulum resident kinase PERK and mediates elF-2alpha dephosphorylation by the gamma (1)34.5 protein. J Virol 2005; 79: 1379-1388.

16. Isler JA, Skalet AH, Alwine JC. Human cytomegalovirus infection activates and regulates the unfolded protein response. J Virol 2005; 79: 6890-6899.

17. Yu CY, Hsu YW, Liao CL, Lin YL. Flavivirus infection activates the XBP1 pathway of the unfolded protein response to cope with endoplasmic reticulum stress. J Virol 2006; 80: 11868-11880.

18. Dediego ML, Nieto-Torres JL, Jimenez-Guardeno JM, Regla-Nava JA, Alvarez E, Oliveros $\mathrm{JC}$ et al. Severe acute respiratory syndrome coronavirus envelope protein regulates cell stress response and apoptosis. PLoS Pathog 2011; 7: e1002315.

19. Tardif KD, Mori K, Kaufman RJ, Siddiqui A. Hepatitis $C$ virus suppresses the IRE1-XBP1 pathway of the unfolded protein response. J Biol Chem 2004; 279: 17158-17164.

20. Trujillo-Alonso V, Maruri-Avidal L, Arias CF, Lopez S. Rotavirus Infection Induces the Unfolded Protein Response of the Cell and Controls It through the Nonstructural Protein NSP3. J Virol 2011; 85: 12594-12604.

21. Ambrose RL, Mackenzie JM. West Nile virus differentially modulates the unfolded protein response to facilitate replication and immune evasion. $J$ Virol 2011; 85: 2723-2732.

22. Umareddy I, Pluquet O, Wang QY, Vasudevan SG. Chevet E, Gu F. Dengue virus serotype infection specifies the activation of the unfolded protein response. Virol J. 2007; 4: 91

23. Zsak L, Lu Z, Kutish GF, Neilan JG, Rock DL. An African swine fever virus virulenceassociated gene NL-S with similarity to the herpes simplex virus ICP34.5 gene. $J$ Virol 1996; 70: 8865-8871.

24. Netherton CL, Parsley JC, Wileman T. African swine fever virus inhibits induction of the stress-induced proapoptotic transcription factor CHOP/GADD153. J Virol 2004; 78: 10825-10828.

25. Zhang F, Moon A, Childs K, Goodbourn S, Dixon LK. The African swine fever virus DP71L protein recruits the protein phosphatase 1 catalytic subunit to dephosphorylate elF2alpha and inhibits $\mathrm{CHOP}$ induction but is dispensable for these activities during virus infection. $J$ Virol 2010; 84: 10681-10689.

26. Ron D, Walter P. Signal integration in the endoplasmic reticulum unfolded protein response. Nat Rev Mol Cell Biol 2007; 8: 519-529.

27. Delom F, Emadali A, Cocolakis E, Lebrun JJ, Nantel A. Chevet E. Calnexin-dependent regulation of tunicamycin-induced apoptosis in breast carcinoma MCF-7 cells. Cell Death Differ 2007; 14: 586-596.

28. Nguyen M, Breckenridge DG, Ducret A, Shore GC. Caspase-resistant BAP31 inhibits fas-mediated apoptotic membrane fragmentation and release of cytochrome $\mathrm{C}$ from mitochondria. Mol Cell Biol 2000; 20: 6731-6740.

29. Harding HP, Calfon M, Urano F, Novoa I, Ron D. Transcriptional and translational control in the Mammalian unfolded protein response. Annu Rev Cell Dev Biol 2002; 18: 575-599.

30. Oyadomari S, Mori M. Roles of CHOP/GADD153 in endoplasmic reticulum stress. Cell Death Differ 2004; 11: 381-389.
31. Calfon M, Zeng H, Urano F, Till JH, Hubbard SR, Harding HP et al. IRE1 couples endoplasmic reticulum load to secretory capacity by processing the XBP-1 mRNA. Nature 2002; 415: 92-96.

32. Nadanaka S, Yoshida H, Kano F, Murata M, Mori K. Activation of mammalian unfolded protein response is compatible with the quality control system operating in the endoplasmic reticulum. Mol Biol Cell 2004; 15: 2537-2548.

33. Okada T, Haze K, Nadanaka S, Yoshida H, Seidah NG, Hirano Y et al. A serine protease inhibitor prevents endoplasmic reticulum stress-induced cleavage but not transport of the membrane-bound transcription factor ATF6. J Biol Chem 2003; 278: 31024-31032.

34. Carrascosa AL, Bustos MJ, Nogal ML, Gonzalez de Buitrago G, Revilla Y. Apoptosis induced in an early step of African swine fever virus entry into vero cells does not require virus replication. Virology 2002; 294: 372-382.

35. Fernando P, Kelly JF, Balazsi K, Slack RS, Megeney LA. Caspase 3 activity is required for skeletal muscle differentiation. Proc Natl Acad Sci USA 2002; 99: 11025-11030.

36. Wurzer WJ, Planz O, Ehrhardt C, Giner M, Silberzahn T, Pleschka S et al. Caspase 3 activation is essential for efficient influenza virus propagation. Embo J. 2003; 22: 2717-2728.

37. Andres G, Garcia-Escudero R, Simon-Mateo C, Vinuela E. African swine fever virus is enveloped by a two-membraned collapsed cisterna derived from the endoplasmic reticulum. J Virol 1998; 72: 8988-9001.

38. Groenendyk J, Michalak M. Endoplasmic reticulum quality control and apoptosis. Acta Biochem Pol 2005; 52: 381-395.

39. Ellgaard L, Molinari M, Helenius A. Setting the standards: quality control in the secretory pathway. Science 1999; 286: 1882-1888.

40. Rivera J, Abrams C, Hernaez B, Alcazar A, Escribano JM, Dixon L et al. The MyD116 African swine fever virus homologue interacts with the catalytic subunit of protein phosphatase 1 and activates its phosphatase activity. J Virol 2007; 81: 2923-2929.

41. Hernaez B, Escribano JM, Alonso C. Visualization of the African swine fever virus infection in living cells by incorporation into the virus particle of green fluorescent protein-p54 membrane protein chimera. Virology 2006; 350: 1-14.

42. Enjuanes L, Carrascosa AL, Moreno MA, Vinuela E. Titration of African swine fever (ASF) virus. J Gen Virol 1976; 32: 471-477.

43. Livak KJ, Schmittgen TD. Analysis of relative gene expression data using real-time quantitative PCR and the 2(-Delta Delta C(T)) Method. Methods 2001; 25: 402-408.

Cell Death and Disease is an open-access journal published by Nature Publishing Group. This work is licensed under the Creative Commons Attribution-NonCommercial-No Derivative Works 3.0 Unported License. To view a copy of this license, visit http://creativecommons.org/licenses/by-nc-nd/3.0/ 